\title{
Semi-harmonic and intermittent solar decimetric spikes
}

\author{
Francisco C. R. Fernandes ${ }^{1}$, Maurício J. A. Bolzan ${ }^{1}$, Reinaldo R. \\ Rosa $^{2}$, José A. S. S. Dutra ${ }^{1}$, José R. Cecatto ${ }^{2}$, Hana Mészárosová ${ }^{3}$ \\ and Hanumant S. Sawant ${ }^{2}$ \\ ${ }^{1}$ Instituto de Pesquisa e Desenvolvimento, Universidade do Vale do Paraiba - UNIVAP, \\ São José dos Campos, SP, Brazil \\ email: guga@univap.br \\ ${ }^{2}$ Divisão de Astrofisica, Instituto Nacional de Pesquisas Espaciais - INPE, \\ São José dos Campos, SP, Brazil \\ email: reinaldo@lac.inpe.br \\ ${ }^{3}$ Ondrejov Observatory, Astronomical Institute Academy of Sciences of the Czech Republic \\ Ondrejov, Czech Republic \\ email: hana@asu.cas.cz
}

\begin{abstract}
We present the analysis of the narrowband solar radio spike emissions observed by the Brazilian Solar Spectroscope (BSS) on 24 June, 1999, at 16:53:55-16:56:48 UT. The data were recorded with temporal resolution of $50 \mathrm{~ms}$ and frequency resolution of $5 \mathrm{MHz}$. The dynamic spectra of clusters of spikes were recorded in 100 channels in the 1.2-1.7 GHz frequency band. The groups of radio spikes observed clearly show spectral semi-harmonic structures and an intermittent temporal patter. The observational parameters were determined from the dynamic spectra analysis. The wavelet analysis technique able us to determine the temporal cadence for the clusters. The frequency ratio between two clusters of spikes recorded at same time were inferred. The preliminary results show average cadence of clusters of spikes occurrence of about 32 seconds and a semi-harmonic average frequency ratio of the order of 1:1.2.
\end{abstract}

Keywords. Sun: activity, radio radiation; decimetric spikes; instrumentation: spectrographs

\section{Introduction}

Observations and analysis of clusters of millisecond spikes exhibiting harmonic bands have been reported by Benz \& Güdel (1987), Güdel (1990), and Krucker \& Benz (1994). Benz \& Güdel (1987) found a semi-harmonic frequency ratio between two spectral peaks of about 1:1.39. Schwarz et al. (1993) found frequency ratio raging between 1:1.03 and 1.3. Güdel (1990) reported a large sample of events showing only integer ratios of 2, 3, 4 and 5. The identifications of harmonic structures and their characteristics can contribute to improve the understanding of the emission mechanisms. According to Wu et al. (1985), such simultaneous emission at multiple harmonic with comparable intensity can be hardly explained by loss-cone distribution, but can be generated by mildly relativistic beam or high-relativistic synchrotron maser (Louarn et al., 1987). The investigations of the semiharmonic structures in spike clusters recorded by the Brazilian Solar Spectroscope (BSS) is on going, including the application of wavelet techniques.

\section{Spikes observations}

Frequently, the spikes form clusters in frequency and time domains, identified at radio dynamic spectra. However, usually, the clusters do not show related behavior 


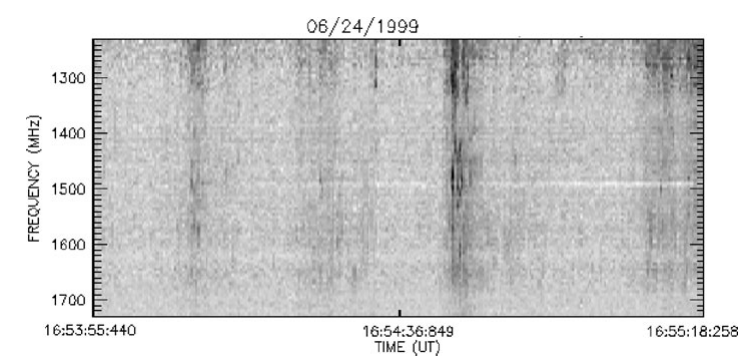

Figure 1. Dynamic spectra of decimetric spikes recorded in 24 June, 1999 ( 16:53:55 UT).

between each other or do not occur simultaneously. Some clusters of decimetric spikes were recorded by BSS in 24 June, 1999, in the time interval of 16:53:55-16:56:48 UT. The data were recorded in 100 frequency channels, equally spaced in the frequency range of $1230-1730 \mathrm{MHz}$, with time resolution of 50 milliseconds and frequency resolution of $5 \mathrm{MHz}$. The dynamic spectra of radio spikes clusters, shown in the Figure 1, indicated splitting in frequency and intermittent behavior.

\section{Wavelet Analysis}

According to Morettin (1999), wavelet transform breaks a signal into components formed by dilated and shifted versions of an original wavelet, called mother wavelet. Given a mother wavelet $\psi(\mathrm{t})$, the dilated and translated daughter wavelets are given by

$$
\psi_{a, b}(t)=\frac{1}{\sqrt{a}} \psi\left(\frac{t-b}{a}\right),
$$

where $a>0$ is the scaling factor controlling the dilation of the mother wavelet and $b$ is the translation parameter determining the shift of the mother wavelet. The scaling factor is directly related to the frequency, or period. The coefficients for the continuous wavelet transform are given by

$$
W(a, b)=\frac{1}{\sqrt{a}} \int_{-\infty}^{\infty} H(t) \psi\left(\frac{t-b}{a}\right) d t .
$$

In the wavelet analysis, the mother wavelet was chosen as Gaussian Morlet given by

$$
\psi(t)=\pi^{-1 / 4} e^{i \omega_{o} t} e^{-t^{2} / 2},
$$

where $\omega_{0}$ is the frequency parameter that allows one to shift the frequency range for investigations.

\section{Results}

We used this wavelet transform to decompose each time-series of spikes clusters (100 frequency channels) into the time and frequency domains. The Figure 2 shows the results of the wavelet analysis for the frequency channel of $1245 \mathrm{MHz}$. The figure shows the average cadence of clusters of spikes is of $32( \pm 2)$ seconds and its harmonics of 16 and 64 seconds. Some clusters of spikes indicated splitting in frequency. The frequency ratio between the mean frequencies of clusters in two simultaneous bands (upper and lower), was estimated for many distinct times. The average frequency ratio is 1:1.2 $( \pm 0.3)$. 

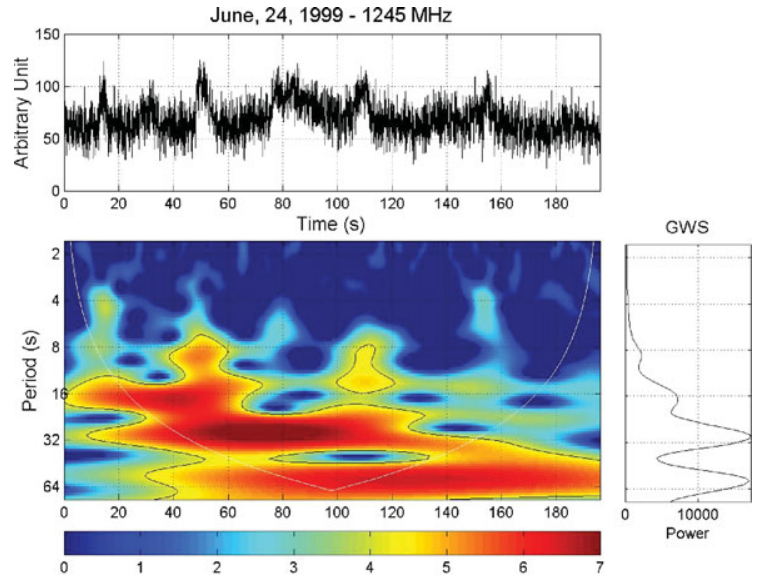

Figure 2. The top panel shows the spikes time evolution for $1245 \mathrm{MHz}$ channel. The wavelet analysis output for this channel is given in the bottom panel.

\section{Concluding Remarks}

Many emission mechanisms have been proposed for the decimetric millisecond spikes bursts: electron-cyclotron maser emission, synchrotron maser emission, plasma emission excited by electrostatic and electromagnetic waves or current-driven direct radiation, according to Aschwanden \& Güdel (1990) (see reviews by Wu et al., 2007; Benz, 1986 and Fleishman \& Melnikov, 1998).

The results obtained to the analysis of the clusters of spikes can support the investigations about their emission mechanisms. In case of the semi-harmonic spikes, according to Benz \& Güdel (1987), their observations cannot be explained considering two integer multiples of the gyrofrequency. However, the maser emission occurs at frequencies slightly different of the harmonics of the gyrofrequency and the radio emission at semi-harmonic frequencies can be generated. Also, the intermittent behavior of the clusters, with of about 32 seconds could be interpreted in terms of magnetic field modulation by MHD waves (Zhao et al., 1990). The detailed analysis of non-harmonic and intermittent decimetric spikes and the implication of their detection to the emission mechanisms, briefly mentioned above, is given by Fernandes et al. (2010).

\section{Acknowledgements}

The authors acknowledge the Brazilian agencies CNPq $(480045 / 2008)$ and FAPESP (06/55883-0).

\section{References}

Aschwanden, M. J. \& Güdel, M. 1990, Solar Physics, 30, 490

Benz, A. O. 1986, Solar Physics, 104, 99

Benz, A. O. \& Güdel, M. 1987, Solar Physics, 111, 175

Fleishman, G. D. \& Melnikov, V. F. 1998, Physics-Uspekhi, 41(12), 1157

Fernandes, F. C. R., Bolzan, M. J. A., Rosa, R. R., Dutra, J. A. S. S., Mészárosová, H., \& Sawant, H. S. 2010, $A \mathscr{E} A$ (in preparation)

Güdel, M. 1987, $A \mathscr{E} A$, 245, L1

Krucker, S. \& Benz, A. O. 1994, A\& A, 285, 1038

Louarn, P., Le Queau, D., \& Roux, A. 1987, Solar Physics, 111, 201

Morettin, P. A. 1999, EdUSP, São Paulo, 1, 42

Schwazs, U., Benz, A. O., Kurths, J., \& Witt, A. 1993, A\&A A, 277, 215

Wu, D. J., Huang, J., Tang, J. F., \& Yan, Y. H. 2007, ApJ, 665, L171

Zhao, R.-Y., Jin, S.-Z., Fu, Q.-J., \& Li, X.-C. 1990, Solar Physics 130, 151 